\title{
Deciphering Tumor Heterogeneity in Hepatocellular Carcinoma (HCC)-Multi-Omic and Singulomic Approaches
}

\author{
Renumathy Dhanasekaran, MD ${ }^{1}$ \\ ${ }^{1}$ Department of Medicine, Gastroenterology and Hepatology, \\ Stanford University School of Medicine, Stanford, California \\ Semin Liver Dis 2021;41:9-18.
}

\begin{abstract}
Address for correspondence Renumathy Dhanasekaran, MD, Department of Medicine, Gastroenterology and Hepatology, Stanford University School of Medicine, 300 Pasteur Drive, Alway Building M211, Stanford, CA 94305-5151 (e-mail: dhanaser@stanford.edu).
\end{abstract}

\begin{abstract}
Tumor heterogeneity, a key hallmark of hepatocellular carcinomas (HCCs), poses a significant challenge to developing effective therapies or predicting clinical outcomes in HCC. Recent advances in next-generation sequencing-based multi-omic and single cell analysis technologies have enabled us to develop high-resolution atlases of tumors and pull back the curtain on tumor heterogeneity. By combining multiregion targeting sampling strategies with deep sequencing of the genome, transcriptome, epigenome, and proteome, several studies have revealed novel mechanistic insights into tumor

Keywords

- HCC

- genomics

- single cell

- heterogeneity

- liver cancer initiation and progression in HCC. Advances in multiparametric immune cell profiling have facilitated a deeper dive into the biological complexity of HCC, which is crucial in this era of immunotherapy. Moreover, studies using liquid biopsy have demonstrated their potential to circumvent the need for tissue sampling to investigate heterogeneity. In this review, we discuss how multi-omic and single-cell sequencing technologies have advanced our understanding of tumor heterogeneity in HCC.
\end{abstract}

Hepatocellular carcinoma (HCC) is a highly lethal malignancy and is a leading cause of cancer mortality around the world, causing around 700,000 deaths annually. ${ }^{1}$ In the U.S., overall cancer-related mortality dropped by an impressive $29 \%$ between 1991 and $2017 .^{2}$ While the 5-year survival rate for all cancers, when combined, improves to $67 \%$, patients with HCC continue to have a dismal 5-year survival of around $18 \%{ }^{2}$ Moreover, despite advances in locoregional therapies, targeted therapies, and immunotherapy, the median survival for advanced HCC remains less than 2 years. One of the factors contributing to the persistent poor survival rates for HCC is the lack of adequate response to conventional therapies.

After the initial promising approval of tyrosine kinase inhibitor sorafenib for advanced HCC in 2007, several clinical trials for HCC evaluating other kinase inhibitors like sunitinib and brivanib failed over the past decade., ${ }^{3,4}$ We have recently seen the additional approval of lenvatinib as first-line therapy, and regorafenib, cabozantinib, and ramucirumab as second-line therapies. ${ }^{5}$ Promising results from a recent phase III clinical trial of combination of immunotherapy (atezolizumab) with bevacizumab indicate this combination will now finally displace sorafenib as first-line therapy for advanced HCC. ${ }^{3}$ Despite these results being considered a major breakthrough for HCC, we have to acknowledge that the combination therapy only marginally improves progression-free survival by around 2 months when compared with sorafenib. Clearly, there is an urgent need to develop better therapies for HCC and in order to do that we first have to understand the reasons for therapy resistance. One of the crucial factors which poses a major challenge not just for developing new therapies but also for identifying novel biomarkers or for predicting response to therapy, is tumor heterogeneity. Hence, deciphering tumor heterogeneity is pivotal to improve clinical outcomes for patients with HCC.

\section{Genomic Landscape of HCC}

Next-generation sequencing (NGS) technologies have steadily evolved over the past 15 years allowing us to sequence an 
exponential number of biological samples, at progressively lower costs. For instance, the cost of sequencing the human genome has fallen from $\$ 10,000$ per megabase of deoxyribonucleic acid (DNA) in 2001, to less than 1 cent per megabase in $2019 .^{6}$ In the pre-NGS era, classical experimental schemes were generally hypothesis-based, where investigations with appropriate controls were designed to either confirm or refute a hypothesis. This approach, while incrementally fruitful, led to a myopic view of biological complexity, since hypotheses are generally limited by the need for a priori knowledge. The application of nonhypothesis-driven approaches using NGSbased technologies to sequence whole cancer genomes has raised the veil on the vast underlying complexity of genetic and epigenetic alterations in cancer cells, thus ushering in an era of rapid new discoveries.

A classic example of NGS-based discovery is the identification of the TERT promoter mutation. Telomerases are enzymes which maintain telomere length of chromosomes during rapid cell division and were known to be upregulated in cancers, endowing cancer cells with immortality. ${ }^{7}$ But the mechanisms by which cancer cells increased telomerase expression were not fully understood. NGS-based whole genome analysis of familial malignant melanoma in 2013 revealed, for the first time, recurrent mutations in the TERT gene promoter, which was associated with higher TERT messenger ribonucleic acid (RNA) levels. ${ }^{8}$ Subsequently, this TERT promoter mutation has been found to be one of the most common genetic events in multiple cancers including HCC. ${ }^{9}$ Several large-scale studies have revealed the genomic landscape of HCC and have identified major somatic mutations, copy number variations (CNVs), and transcriptomic signatures of HCC. ${ }^{9-13}$ Few of the most common somatic events in HCC include mutations in the TERT promoter, TP53, CTNNB1, AXIN1, or ARID1A genes and most common CNV involve MYC, MET, CCND1, VEGFA, and FGF19 genes. Mutational signature patterns have also helped in identifying etiologic agents which drive cancer initiation, like signatures associated with tobacco, aflatoxin, or aristolochic acid exposure. ${ }^{9,14}$ Thus, the application of NGS-based sequencing has rapidly led to expansion of our knowledge of cancer biology thus opening the door to further questions and research. But it has to be noted that the aforementioned NGS studies have identified genetic events mostly by sequencing bulk tumors and hence do not capture the heterogeneity in HCC.

\section{Cancer Heterogeneity in Hepatocellular Carcinoma}

HCC is known to exhibit exceptional heterogeneity compared with other tumors. Tumor heterogeneity can mean different things in different contexts-(1) etiologic - HCC can be caused by varied risk factors ranging from viral hepatitis to nonalcoholic fatty liver disease to toxins, (2) geographic heterogeneity - HCC has different incidence and outcomes in different regions of the world, or (3) molecular heterogeneity. In this review, we will primarily be discussing molecular heterogeneity, which refers to the variations in genetic events, gene expression patterns, activated pathways, im- mune infiltrates, or stromal changes across tumors. Molecular heterogeneity can be further classified into-(1) interpatient - molecular differences between tumors of different patients, (2) intertumor - refers to variations between different tumors in the same patient, or (3) intratumor heterogeneity refers to variations across different regions of an individual tumor in the same patient (-Fig. 1). One of the main reasons for the significant tumor heterogeneity seen in HCCs is that they generally arise in the background of a highly inflamed and fibrotic procarcinogenic microenvironment created by cirrhosis, which exerts a field effect throughout the liver. Hence, synchronous and metachronous tumor initiating events occur concurrently all over the liver, leading to significant inter- and intratumor heterogeneity.

Early studies elegantly uncovered HCC heterogeneity by sampling multiple nodules from individual patients and studying them using platforms such as immunohistochemistry (IHC), Sanger sequencing, Southern blot hybridization, or DNA fingerprinting. ${ }^{15-17}$ However, recent advances in genomics and single-cell sequencing have opened the floodgates of cancer research and have provided a rare window into the molecular heterogeneity in HCC. In this review, we will discuss how multi-omic and single-cell sequencing technologies have enabled us to navigate and understand tumor heterogeneity in HCC.

\section{Multi-Omic Approaches to Tumor Heterogeneity in HCC}

NGS-based multi-omic analyses have been particularly important in studying tumor heterogeneity. Understanding and quantifying tumor heterogeneity is crucial since it has several translational implications for predicting recurrence and response to therapy. Tumors with high levels of heterogeneity generally do not respond well to therapies since the selective pressure introduced by the therapy leads to expansion of resistant subclones or to the emergence of new drug-tolerant clones. Most of the genomic studies have analyzed sequencing data obtained from bulk tumor tissue leading to limited understanding of the tumor heterogeneity. Novel platforms such as single-cell sequencing, mass cytometry, and spatial transcriptomics are now enabling us to decode the complexity of the tumor microenvironment at a very high resolution. Using these platforms along with serial sampling and multiregion tumor sampling is allowing us to develop a deeper understanding of the temporal and spatial heterogeneity of HCC (-Fig. 2).

A malignant tumor is not just a mass of rapidly proliferating tumor cells but is a highly complex quasi-organ which is infiltrated by a plethora of host immune cells, and stromal cells, is supported by an extracellular matrix and also entwined by blood vessels which provide oxygen and nutrients. ${ }^{18}$ The heterogeneity present in tumors does not necessarily arise solely from the cancer cells themselves but also from variations in the immune cells, stromal cells, and other components of the microenvironment (TME) (-Fig. 1). By extracting and studying single cells from tumors, we are now able to 


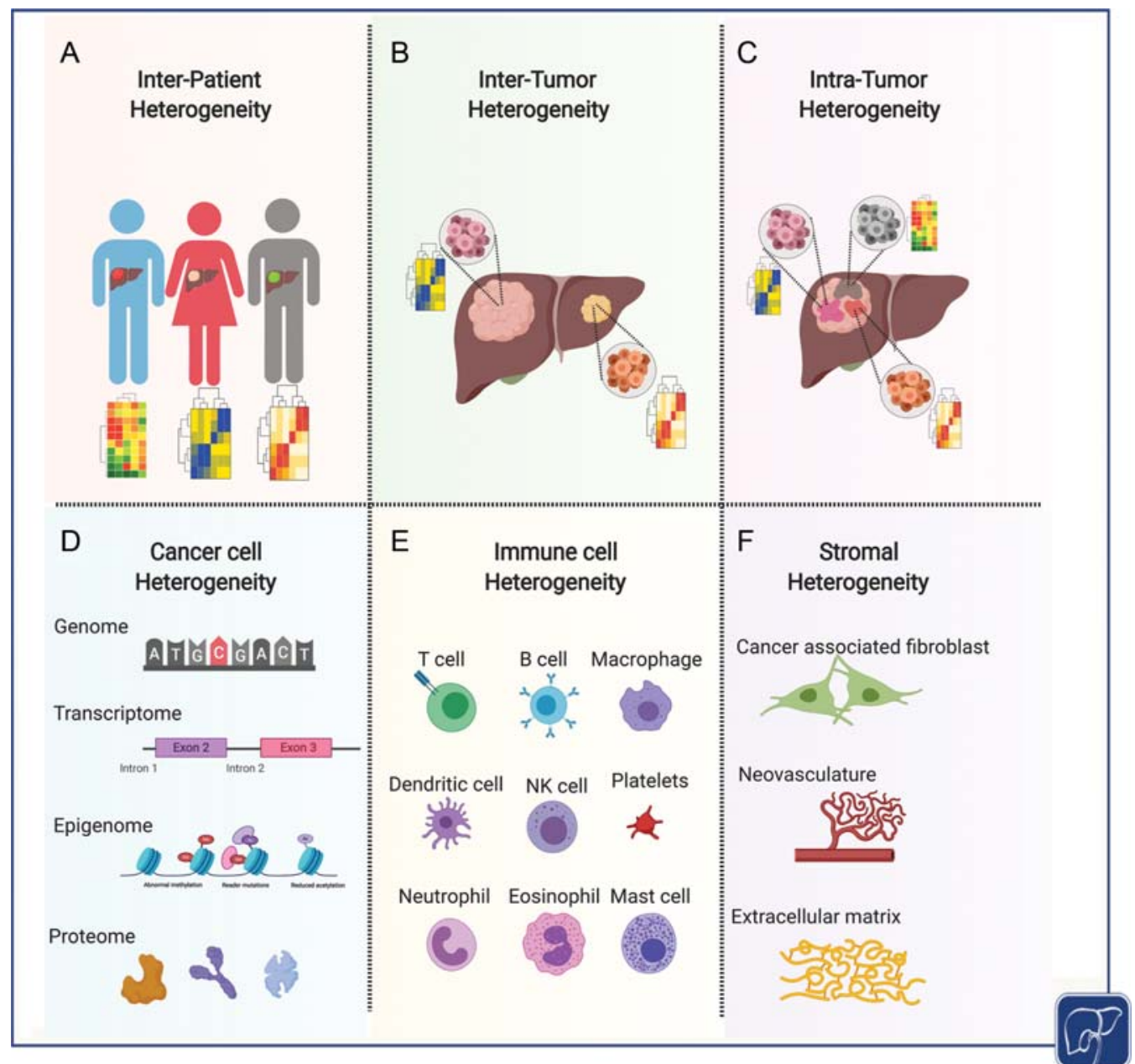

Fig. 1 Types and sources of tumor heterogeneity in hepatocellular carcinoma (HCC). The top panel present the different types of heterogeneity in HCC. (A) Interpatient - molecular differences between tumors of different patients, (B) intertumor - variations between different tumors in the same patient, and $(C)$ intratumor heterogeneity refers to variations across different regions of an individual tumor in the same patient. The bottom panel lists the different sources of heterogeneity in HCC which can result either from - (D) cancer-cell intrinsic variations, (E) differences in tumor immune cell infiltrates, and (F) changes in tumor stroma.

independently understand the heterogeneity in these discrete compartments of the TME. We will discuss below the studies exploring heterogeneity either in the cancer cells or in the immune cell compartment.

\section{Multi-Omic Analysis of Cancer Cell Heterogeneity in HCC}

Cancer cells exhibit high degree of genomic instability and are hence the main source of heterogeneity in a tumor, we will address them first. To study heterogeneity using bulk tumor sample sequencing, investigators generally sample multiple discrete regions from the same tumor or multiple regions from different tumors in the same patient and compare them using this multiregion targeting strategy.

For instance, Ling et al used laser dissection microscopy to perform honeycomb-like precise microdissections of a single 35-mm HCC tumor and extracted DNA from 286 separate regions for genotyping, and performed whole exome sequencing of 23 of these dissected regions. Each sample contained approximately 20,000 cells. $^{19}$ Surprisingly, they found extreme genetic diversity in this tumor with more than 100 million estimated coding region mutations, and 6 to 7 major clones. The 23 exome sequenced samples within this tumor displayed 20 unique cell subclones. Authors analyzed these data by modern population genetic theory and concluded that the extreme genetic diversity implies evolution under the nonDarwinian mode. This theory possibly explains the high degree of heterogeneity noted in HCC. Moreover, we can easily see how the prevalence of genetic driver events can be underestimated when we sample and sequence only one site per tumor for each patient.

In another interesting study, Shi et al performed multiregional whole exome sequencing of two synchronous HCCs and one cholangiocarcinoma nodule from the same patient and found that the three primary lesions showed almost no overlapping mutations or CNVs underscoring intertumor heterogeneity in this patient. ${ }^{20}$ Moreover, this patient developed postoperative recurrence and sampling the two recurrent nodules showed that their mutational profile was concordant with just one of the primary HCC nodules, not the other two, indicating their origin. This patient had underlying hepatitis $B$ 


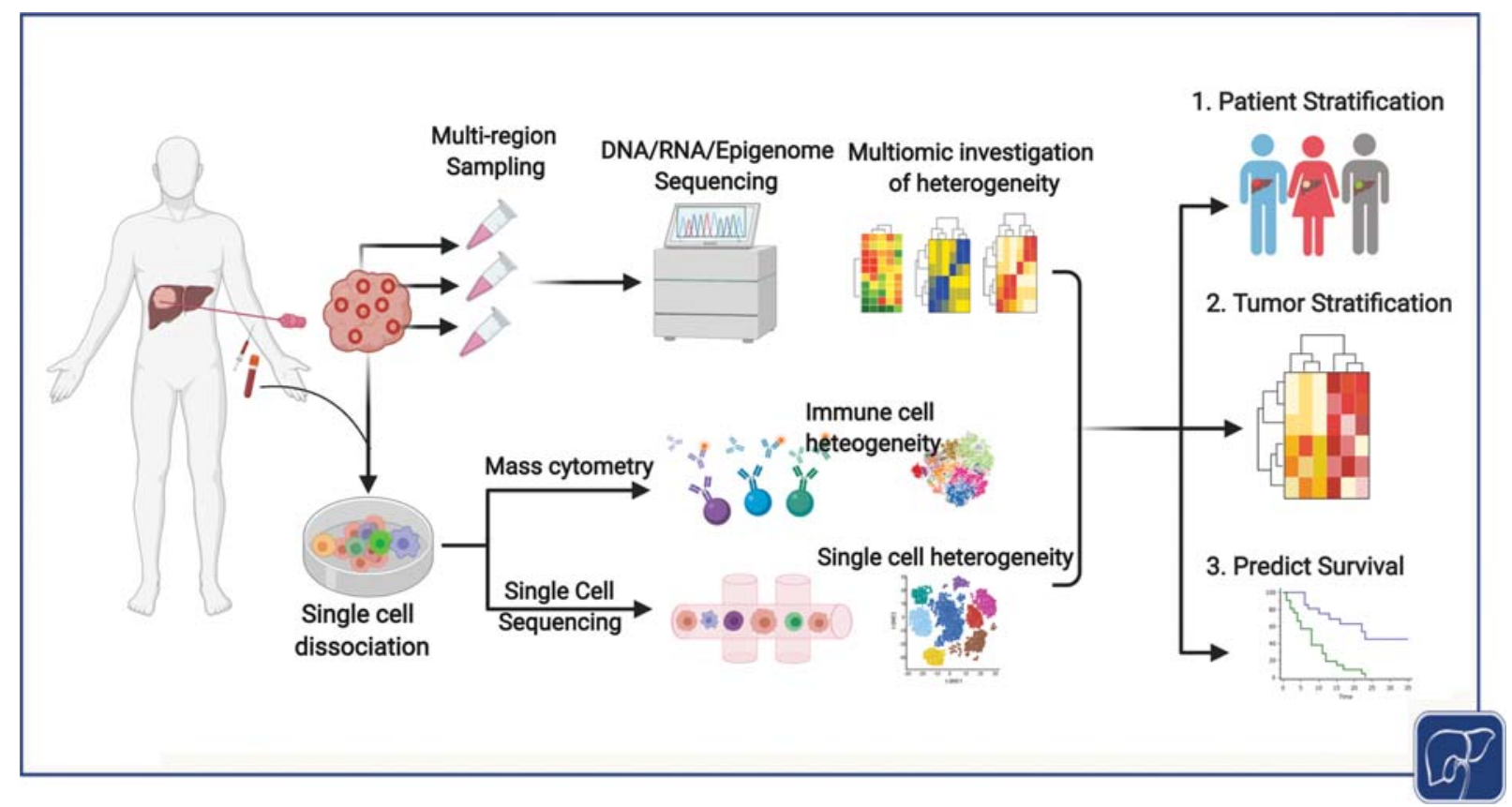

Fig. 2 Multi-omic approaches to study tumor heterogeneity in hepatocellular carcinoma (HCC). Multiregion tumor sampling or serial liquid biopsy sampling can be used to study tumor heterogeneity. Next-generation sequencing (NGS)-based sequencing technology, single-cell sequencing, mass cytometry, mass spectrometry, or other high-resolution methods can be used to study the extracts from multiregion sampling and dissociated single cells decipher tumor heterogeneity. This knowledge can then be used to stratify patients and tumors or to predict clinical outcome.

virus (HBV)-related cirrhosis possibly explaining the emergence of these synchronous but disparate primary liver cancer lesions. HCC induced by HBV can show high degrees of intertumor heterogeneity since HBV viral integration into the host genome is an added driver of carcinogenesis in this subset of HCCs. Xue et al studied 43 separate lesions from 10 patients with multifocal HBV-HCC using exome sequencing. ${ }^{21}$ They found that the degree of intratumoral heterogeneity varied widely between patients with the proportion of ubiquitous mutations ranging from 8 to $97 \%$ of all mutations. While satellite nodules near the main nodule appeared to mostly have shared genetic events with the primary, metastatic nodules or even tumor thrombus appeared to have distinct genetic events providing evidence for clonal evolution during tumor progression. On construction of phylogenetic trees, they found that HBV integrations in driver genes like MLL4 or TERT occurred both in the trunks and branches of the tree. These results suggest that viral integrations may occur either early or late during tumor evolution and play an important role in driving tumor heterogeneity in HCC.

While most studies have explored the exome sequencing profile or the transcriptome to study tumor heterogeneity, Buczak et al evaluated heterogeneity using large-scale proteomics. ${ }^{22}$ This approach is valuable since analyzing the proteomic data enables us to gain functional insights into the heterogeneity introduced by the genetic events. Buczak et al used a mass spectrometry-based proteomic approach to explore inter- and intratumor spatial heterogeneity in five patients with $\mathrm{HCC}^{22}$ Interestingly, authors also explored spatial heterogeneity in HCC by carefully comparing the proteomic profile of tumor cells microdissected from the center of the tumor versus those from the periphery, rather than performing random sampling. The small number of samples did not allow them to make robust conclusions, but the study did demonstrate prominent interpatient variability in the differential proteomic expression between the cells at the center of the tumor and those at the periphery. For example, mitochondrial metabolism appeared to be downregulated in the periphery in multiple samples, compared with the center. This study illustrates the added complexity of tumor cell spatial location on functional heterogeneity.

\section{Multi-Omic Analysis of Immune Cell Heterogeneity in HCC}

Tumor heterogeneity can arise from the infiltrating host immune and stromal cells and not just from the cancer cells themselves. With recent promising results for immunotherapy in HCC, there is increasing interest in understanding the specific immune subsets present in the tumor microenvironment and how they interact with the cancer cells. Classic approaches like multiplex IHC and flow cytometry have previously revealed immune cell heterogeneity and helped identify specific subtypes based on immune infiltrates. For instance, Kurebayashi et al used multiplex immune cell IHC to evaluate 919 regions from 158 resected HCC specimens and were able to classify the HCCs into-immune-high, immune-mid, or immune-low subtypes, with the immune-high subtype having a generally better prognosis. ${ }^{23}$ More recently technologies such as mass cytometry, codetection by indexing, and T cell receptor (TCR) sequencing are allowing us to directly interrogate the tumor immune microenvironment more comprehensively. ${ }^{24-26}$ 
Combining multi-omic sequencing with immune profiling is a powerful strategy to gain a more global overview into tumor complexity. Losic et al pursued this strategy by integrating DNA-, RNA-, and TCR-sequencing from 71 spatially discrete regions from the same tumor-derived 14 human liver cancer specimens to investigate intratumoral heterogeneity. ${ }^{27}$ They found significant heterogeneity not just in tumor-specific antigen expression in different regions of a tumor, but also in immune infiltrate burden and immune cell clonality. They also found that the regional clonal immune response contributes significantly to intratumoral heterogeneity in HCC. To understand this data at a higher resolution, they followed this up by performing single-cell sequencing of around 20,000 cells from two geographically distant regions and confirmed significant heterogeneity in the transcriptional pathways activated in distant regions within the same nodule. ${ }^{27}$

In another study which used similar multi-omic approaches, the investigators performed DNA-sequencing, RNA-sequencing, mass spectrometry-based proteomics, and also mass cytometry for immune profiling of 42 discrete samples from eight HCC patients. ${ }^{28}$ They found significant intralesional heterogeneity in tumor cells at all levels ranging from the genome to the proteome. Compared with the cancer cells, the immune infiltrates in HCC appeared to be less heterogeneous and they were able to cluster all tumors into three groups based on the immune profile-subtype 1 with competent immune response and good prognosis, subtype 2 were "cold" tumors with minimal immune infiltrates, and subtype 3 with higher immunosuppressive infiltrates and exhausted $\mathrm{T}$ cells. ${ }^{28}$ These data suggest that studying the tumor immune microenvironment might be a more tractable approach to predict response to immunotherapy than sequencing just the tumor cells.

Another interesting feature of multifocal HCC is the coexistence of intrahepatic metastasis, which are derived from vascular spread of larger primary tumor, and multicentric primary HCC where the lesions are not related to each other. Dong et al evaluated distinctions between multicentric HCCs and intrahepatic metastasis by combining data from DNAsequencing, RNA-sequencing, multiplex immunostaining, immunopeptidomics, and TCR sequencing of 47 tumors from 15 patients with multifocal HCC. ${ }^{29}$ Authors described significant spatiotemporal differences in the transcriptome and immune profiles between intrahepatic metastasis and multicentric HCCs. They found that metastatic lesions shared neoantigens and TCR repertoires with the primary lesion and had lower T cell infiltration along with higher M2-like macrophage infiltration. However, multicentric HCCs were characterized by higher expression of immune checkpoints and also higher rates of immune editing. These spatiotemporal changes imply that response to immunotherapy will differ between those with intrahepatic metastasis versus multicentric HCC. Moreover, they were able to integrate the data from the sequencing and immune profiling platforms to develop a classifier which could predict recurrence. Thus, large-scale multi-omic data from these studies clearly reveal the vast complexity and heterogeneity in HCC and raise the concern that single-site biopsy from individual tumors might not be enough to risk stratify patients or to make therapeutic decisions.

\section{Tumor Heterogeneity from Other Stromal Cells}

Apart from immune cells, other stromal cells like cancerassociated fibroblasts (CAFs) and endothelial cells also contribute to tumor heterogeneity in HCC. CAFs represent a mixed population of cells which can be derived from local fibroblasts, mesenchymal stem cells, vascular smooth muscle cells, or even from cancer cells. ${ }^{30}$ Multi-omic and single-cell technologies have been successfully used to profile CAFs from breast and lung cancer and they have identified specific functional subtypes of CAF. ${ }^{31,32}$ In general, there appear to be two major phenotypes, a contractile phenotype which contributes to the tumor matrix and an inflammatory phenotype which modulates the local immune response via its secretome. ${ }^{33}$ Vascular endothelial cells are another source of intratumoral heterogeneity. ${ }^{34}$ Ma et al recently used single-cell RNAseq from 19 liver cancer samples and found that the transcriptional states of nonmalignant cells such as tumor endothelial cells and CAFs were reprogrammed in tumors with higher heterogeneity and the gene signature derived from these stromal cells, could be used to discriminate heterogeneity in HCCs. ${ }^{35}$

\section{Single-Cell Sequencing Approaches to Understand Tumor Heterogeneity in HCC}

Most of the NGS-based multi-omic techniques described above like whole exome sequencing or RNA-sequencing typically extract DNA or RNA by lysing bulk tissue. Hence, the data represents the average mutation rate or average gene expression across millions of cells of heterogeneous origin. While this approach has provided valuable insights into heterogeneity, it does obscure the differences in expression between the different kinds of cells thus leading to an underestimation of the biological complexity of cancer. Single-cell sequencing is an approach where bulk tumors are dissociated to isolate single cells, followed by DNA or RNA extraction from these cells which are then sequenced individually. ${ }^{36}$ Advances in microfluidic technology and molecular barcoding have made the profiling of tens of thousands of individual cells not only technically feasible but also costeffective.

Investigators are now increasingly using single-cell sequencing to tractably perform large-scale analysis at a single-cell level in multiple cancers, at an unprecedented resolution. These single-cell sequencing technologies are revolutionizing our ability to understand cancer and opening a window into cancer heterogeneity. Single-cell DNA sequencing enables us to define the mutation profile of individual subclones within tumors and to understand their evolutionary trajectory. Meanwhile, single-cell RNA sequencing allows us to evaluate the transcriptional profile of individual cancer cells in comparison to the infiltrating stromal cells or host immune cells. Other technologies like single-cell DNA methylome sequencing and single-cell transposase-accessible chromatin with sequencing (ATACseq) promise to reveal novel insights into the epigenetic landscape 
of tumors at a single-cell resolution. We will now highlight the major studies employing these single-cell technologies to study heterogeneity in the cancer cells and host immune cells in HCC.

\section{Single-Cell Genomics of Cancer Cells in HCC}

One of the exciting aspects of singlulomics is the opportunity to study how genetic or epigenetic driver events impact gene expression in an individual cell, thus establishing the functional relevance of the genetic event to cancer progression. Hou et al pursued this by exploring the genome, transcriptome, and methylome of each individual cell using scTrio-seq of 25 single cancer cells derived from a patient with HCC. ${ }^{37}$ Upon hierarchical clustering of these 25 cells they found two subtypes one with copy gains in chromosome 8, 11, and 20; while the second subpopulation showed loss of chromosomes 4 and 16 . They were able to demonstrate that these large-scale CNVs were associated with corresponding gene expression changes, while these CNVs did not correspond with the methylomic changes. Interestingly, they found that clustering based on CNV or methylome sequencing classified the cells into the exact same two clusters. By overlapping these clusters with the gene expression changes, they showed that cells in the first subpopulation with higher $\mathrm{CNV}$ were less responsive to immune recognition as they had downregulation of multiple genes in the acute inflammatory response and complement activation pathways. Thus, the authors used the information from the triple-omics analysis to infer specific biological properties of individual subclones within a tumor at a single-cell level, and demonstrate how these findings may have translational implications in predicting response to immunotherapy.

The study of cancer stem cells (CSCs) has been an area of controversial research and several investigators have identified divergent populations of liver CSCs based on expression of surface markers such as CD44, CD90, CD133, or EpCAM. ${ }^{38}$ Single-cell sequencing is an ideal platform to explore this further, since we are now able to evaluate the expression of a multitude of stem cell markers on each individual cell. ${ }^{39,40}$ Zheng et al used flow cytometry to sort and isolate CSC subpopulations (CD133+/CD24+/EpCAM +) from two HCC cell lines and 118 cells derived from a single patient with HCC who had undergone resection. ${ }^{40}$ Single-cell whole transcriptome sequencing of these cells revealed significant phenotypic and functional heterogeneity in self-renewal capacity and differentiation depending on individual surface marker expression. The transcriptomic heterogeneity and functional diversity observed in the diverse CSC population highlights the potential origin of cancer cell heterogeneity.

One of the direct mechanisms by which HBV causes HCC is via integration of the HBV genome into the host genome eventually leading to molecular transformation of benign hepatocytes into cancer cells. ${ }^{41}$ But several questions remain unanswered, specifically, the frequency of integration essential for hepatocarcinogenesis, the role of spatial heterogeneity in viral integration sites within the liver, and the timing of viral integration during tumor evolution. Duan et al performed single-cell whole genome sequencing to profile 96 cancer cells and 15 normal liver cells collected from 3 patients with hepatitis B-driven $\mathrm{HCC}^{42}$ They showed that all the single cells in a specific HCC clone exhibited the same HBV integration indicating these integrations are early genetic drivers of cancer initiation and remain stable during tumor evolution. Also, tumors with multinodular morphology were typically polyclonal and displayed a high degree of heterogeneity. Thus, this study highlights how different sites of HBV integrations in the host liver cell genome can account for the clonal diversity of multifocal HBV-HCC.

\section{Single-Cell Genomics to Study Immune Cells in HCC}

Another major advantage of single-cell technology is the ability to analyze the expression patterns in host immune cells infiltrating the tumor. Zhang et al combined two single-cell technologies to study $>75,000$ single CD45+ immune cells infiltrating HCC derived from 16 patients with HCC, and compared it to immune cells in the adjacent normal liver, lymph node, blood, or ascitic fluid. ${ }^{43}$ Using single-cell sequencing Zhang et al identified two distinct states of tumorassociated macrophages (TAMs) in human HCC-one with a myeloid-derived suppressor cell-like profile and another with a M2-like TAM profile. Further, they observed that the macrophages with M2-like profile showed expression of genes such as SLC40A1 encoding ferroportin, suggesting that iron metabolism is potentially involved in macrophage polarization in tumors. Next, they used computational lineage tracing technology to reveal potential migration patterns of immune cells from the tumor to distant sites like lymph nodes or ascitic fluid. For instance, they identified a subset of dendritic cells which were LAMP3+ and appeared to be home to hepatic lymph nodes from the tumor and crosstalk with lymphocytes in the TME leading to dysfunctional T cells. This analysis of immune cells from multiple sites provides valuable insight into the dynamic modification of immune cell profiles during tumor evolution and highlights that cancer is not just a local phenomenon but is a systemic disease.

Some investigators have taken the approach to focus primarily on systematically interrogating tumor infiltrating immune cells using single-cell sequencing. Zheng et al performed singlecell RNA sequencing of around 5,000 $\mathrm{T}$ cells isolated from the tumor, adjacent normal liver, and the peripheral blood of six patients with HCC. ${ }^{44}$ The high-resolution data enabled the authors to identify 11 distinct T cell subtypes in HCC. Exhausted CD8 + T cells were the largest cluster of tumor infiltrating CD +8 $\mathrm{T}$ cells and TCR analysis revealed these exhausted T cells had likely evolved from other CD8 $+\mathrm{T}$ cell subtypes during tumor evolution. Another major T cell subset of interest was the HCCspecific CCD8 +/LAYN + regulatory $\mathrm{T}$ cells (Tregs) that were clonally expanded and enriched in the tumor when compared with the nontumorous liver. These Tregs are known to have an immunosuppressive function and their enrichment could thus represent a mechanism by which cancer cells evade tumorspecific immune responses. Thus, the authors were able to use single-cell sequencing to reveal novel subtypes of immune cells in HCC and infer their developmental trajectory. 
Moreover, these data serve as a valuable resource and their analysis can have clinical implications in understanding resistance to immunotherapy. Wang et al reanalyzed the single-cell sequencing data generated by Zheng et al along with independent $\mathrm{T}$ cell flow cytometry data from patients with chronic hepatitis B or HCC, to identify mechanisms for T cell exhaustion. They found that the mechanisms of $\mathrm{T}$ cell exhaustion in chronic hepatitis B without HCC to be distinct from mechanisms responsible for T cell exhaustion in HCC, since the $\mathrm{T}$ cells from these conditions clustered into distinct T cell exhaustion modules. ${ }^{44,45}$

Studies using single-cell analysis have not only provided mechanistic insights into drug resistance in HCC but also help predict clinical outcomes. By analyzing single-cell RNAseq profiles of 19 primary HCC and intrahepatic cholangiocarcinoma patients, it was found that vascular endothelial growth factor (VEGF) signaling in tumors promotes an immunosuppressive microenvironment, thus providing a rationale for combination therapy of immune checkpoint inhibitors with VEGF inhibitors in HCC. ${ }^{35}$ Moreover, they were able to link the degree of intratumoral heterogeneity to patient prognosis, as tumors with higher transcriptomic diversity were associated with patient's worse overall survival. Thus, ongoing studies in single-cell sequencing show significant promise to reveal translationally relevant insights into tumor heterogeneity and therapeutic resistance in HCC.

\section{Using Liquid Biopsy to Study Tumor Heterogeneity}

The analysis of tumors using biomarkers such as circulating tumor cells (CTCs) or cell-free DNA (cfDNA) in peripheral blood has been coined "liquid biopsy" since we are now able to sample tumors noninvasively. ${ }^{46}$ The rapid developments in the field of liquid biopsy research have ushered in a promising phase in biomarker research and liquid biopsy has already shown potential to help us decipher tumor heterogeneity in HCC. ${ }^{47,48}$ During tumor clonal evolution, cancer cells sequentially acquire genetic events throughout the different steps in the metastatic cascade namely invasion, dissemination, and colonization. Spatial heterogeneity between cancer cells inside the tumor versus those in the circulation and those at metastatic sites has been an area of great interest. To study this, Sun et al collected blood either from peripheral vein, peripheral artery, hepatic veins, infrahepatic inferior vena cava, and portal vein before tumor resection in 73 patients with $\mathrm{HCC}^{49}$ Authors found that CTCs were predominantly epithelial at vascular sites proximal to the tumor, but transformed to a mesenchymal-like phenotype during dissemination in the circulation. Tumors that exhibited a high degree of epithelial-mesenchymal transition activity were noted to have a higher number of total CTCs in the hepatic veins. Moreover, CTC and circulating tumor burden were observed to predict postoperative lung metastasis and intrahepatic recurrence, respectively. This study highlights the value of serial and spatial sampling of the peripheral blood in understanding tumor heterogeneity and predicting clinical outcomes in HCC.
cfDNAs are very encouraging biomarkers which have the potential to circumvent existing challenges in tumor sampling. Since cfDNA potentially will contain DNA shed from all the major tumor nodules, they can help identify, and serially follow, the major genomic events across multiple tumor regions without the need for repeated biopsies. Huang et al evaluated the value of cfDNA in tracking tumor heterogeneity. ${ }^{50}$ They performed whole exome sequencing and targeted deep sequencing of 32 multiregional tumor samples along with matched preoperative cfDNA sequencing from five patients with HCC. Authors found that complementing targeted deep sequencing of a single tumor specimen with cfDNA sequencing enabled them to identify genetic variation that could be targeted by Food and Drug Administration (FDA) approved drugs in $37.1 \%$ patients. This data, though preliminary, provides evidence for the utility of cfDNA analysis in evaluating tumor heterogeneity.

\section{Experimental Systems to Model Tumor Heterogeneity}

Our current understanding of tumor heterogeneity is limited by the traditional experimental model systems such as immortalized cell lines or transgenic mouse models. We need to develop in vitro and in vivo novel model systems which can capture the complexity of human HCCs. Patientderived primary cell lines, organoids derived from human HCC samples, and patient-derived xenografts (PDXs) are promising model systems which can be employed to study heterogeneity (-Fig. 3). Tumor samples either from resection or biopsy can be used to establish patient-derived primary cancer cells which can be maintained in culture for extended periods of time and can be used for therapeutic drug screening. ${ }^{51}$ This strategy can be successfully combined with multiregion sampling to model tumor heterogeneity in the dish. Gao et al sampled 55 regions from 10 patients with HCC undergoing curative resection, and isolated primary cancer cells. ${ }^{52}$ They maintained these cells in low-passage culture and performed whole-exome sequencing, copy number analysis, and high-throughput drug screening. They found that cells from four discrete tumor regions containing genetic alterations like FGF19, DDR2, PDGFRA, and TOP1 were sensitive to corresponding targeted therapeutic agents, thus establishing proof-of-principle that such in vitro approaches can be used to study tumor heterogeneity in HCC.

Organoids are preclinical models where cancer cells are cultured in vitro as three-dimensional structures. ${ }^{53}$ Developing organoids from multiregion sampling is a good experimental approach to study tumor heterogeneity. Li et al used this approach and generated 27 liver cancer organoid cell lines by sampling distinct regions of the tumor. ${ }^{54}$ They tested a library of 129 cancer drugs using these organoid cell lines and were able to identify a subset of drugs that appeared pan-effective, thus demonstrating the utility of cancer organoid drug testing in drug discovery pipelines. PDXs are established by growing human HCC xenografts in immunocompromised mice. PDX models have already been successfully used to study tumor progression, identify novel biomarkers, and to perform 


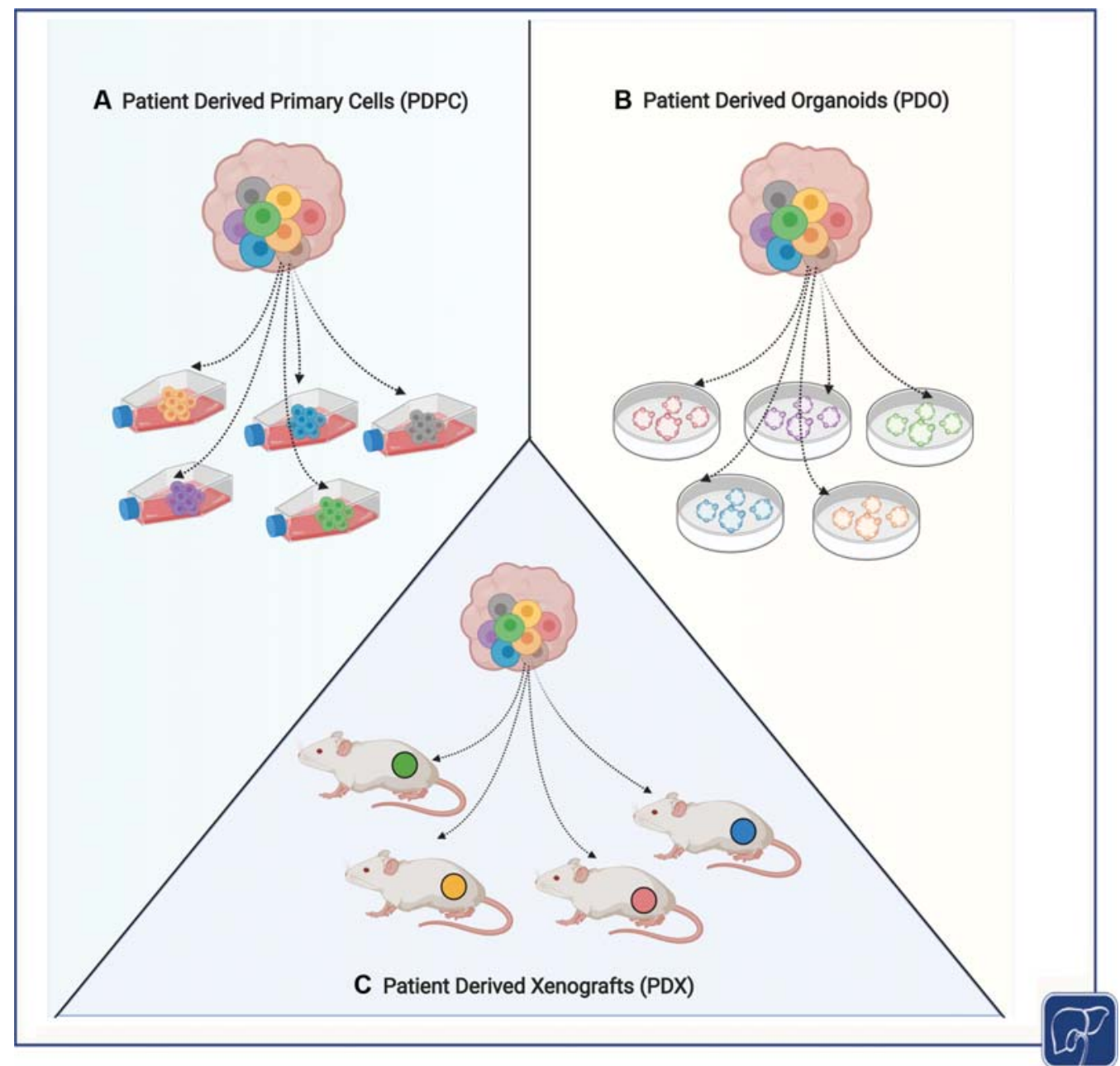

Fig. 3 Experimental model systems to study tumor heterogeneity in hepatocellular carcinoma (HCC). Multiregion tumor targeting can be coupled with tumor dissociation followed by primary cell culture (A) or organoid culture (B) in vitro to develop patient-derived primary cells or patient-derived organoids. These dissociated single cells can also be grown as xenografts in immunocompromised mice to establish patientderived xenografts $(C)$. All these experimental model systems can be used to interrogate tumor heterogeneity and to perform therapeutic drug screens.

preclinical personalized drug screens. ${ }^{55-57}$ Using multiregion sampling strategies to develop PDXs can enable investigators to model intratumor heterogeneity in vivo. While such approaches have been successful in other cancers in combination with other technologies like exome sequencing, ${ }^{58,59}$ establishing PDXs in HCC is challenging since the rate of engraftment is traditionally considered to be low. ${ }^{55}$

\section{Challenges and Future Directions}

The advances in NGS-based multi-omic sequencing and single-cell sequencing have brought tremendous excitement and are finally enabling us to comprehensively study tumor heterogeneity in HCC. But several challenges remain in harnessing these technologies to the fullest. A relatively unique predicament in $\mathrm{HCC}$ is that currently there is no clinical indication to biopsy the tumor to confirm the diagnosis. The resulting lack of access to tissue samples has already served as a major roadblock in studying tumor progression and heterogeneity in HCC. The approval of novel biomarker-stratified therapies will hopefully provide clinical justification for tissue sampling in patients with HCC in the future. Until then, investigators should continue to pursue tissue samples acquisition under research protocols with patient consent. Serial sampling of cfDNA is another promising tool which can help us bypass the need to obtain multiregion tumor sampling to understand heterogeneity. Although early results from cfDNA studies are promising, these still need to be validated in larger cohorts before they can be rolled out to routine clinical care. Moreover, it is unlikely that CTC or cfDNA will fully capture the heterogeneity in the primary tumors both due to sensitivity of the assays and also the likelihood that only a small proportion of the primary tumor cells or tumor DNA are in circulation, thus limiting our capacity to detect subclonal mutations which are present in only a subset of HCC cells.

Lastly, tumor heterogeneity is felt to be a major reason for the failure of multiple drugs in clinical trials for cancer. ${ }^{60,61}$ There is limited direct evidence that heterogeneity leads to therapeutic resistance in HCC, since tumors will have to be biopsied before and after treatment to determine this. ${ }^{61}$ But given the degree of heterogeneity observed in HCC, and the 
experimental data linking heterogeneity to drug resistance in other cancers, there is significant concern that heterogeneity could lead to clinical trial failures in HCC. ${ }^{60,62}$ We need to develop a strategy to account for tumor heterogeneity while enrolling patients with HCC in future clinical trials. Protocol biopsies followed by single-cell sequencing before and after treatment can help us understand the evolutionary trajectory of therapy resistance. Moreover, biomarker-stratified approaches and subtype-enriched enrollment in clinical trials can hopefully allow us to overcome the challenges introduced by tumor heterogeneity.

To conclude, rapid developments in multi-omic and singulomic technologies are enabling us to build quantitative, highresolution maps of tumor landscapes, allowing us to delineate cellular lineage and functions at a single-cell level. These advances allow us to finally realize the full potential of personalized medicine in the care of patients with HCC.

\section{Main Concepts and Learning Points}

- HCCs exhibit high degree of interpatient, intertumor, and intratumor heterogeneity.

- Apart from the cancer cells, immune cells and stromal cells are also drivers of intratumor heterogeneity.

- Multitargeted tumor sampling combined with multiomic sequencing strategies have enabled us to gain deeper insights into tumor heterogeneity in HCC.

- Single-cell sequencing has allowed us to create highresolution maps of the diverse cell populations in a heterogeneous tumor.

- Insights gained from the multi-omic and singulomic study of tumor heterogeneity will help develop novel biomarkers and improve therapeutic targeting of HCC.

\section{Funding}

U.S. Department of Health and Human Services $>$ National Institutes of Health $>$ National Cancer Institute CA222676.

Conflict of Interest

No conflicts of interest to report.

\section{References}

1 Liver Cancer Survival Rates American Cancer Society. Accessed July 24, 2020 at: https://www.cancer.org/cancer/liver-cancer/detection-diagnosis-staging/survival-rates.html

2 Siegel RL, Miller KD, Jemal A. Cancer statistics, 2020. CA Cancer J Clin 2020;70(01):7-30

3 Finn RS, Qin S, Ikeda M, et al;IMbrave150 Investigators. Atezolizumab plus bevacizumab in unresectable hepatocellular carcinoma. N Engl J Med 2020;382(20):1894-1905

4 Faivre S, Rimassa L, Finn RS. Molecular therapies for HCC: looking outside the box. J Hepatol 2020;72(02):342-352

5 Bangaru S, Marrero JA, Singal AG. Review article: new therapeutic interventions for advanced hepatocellular carcinoma. Aliment Pharmacol Ther 2020;51(01):78-89

6 Wetterstrand KA. DNA Sequencing Costs: Data from the NHGRI Genome Sequencing Program (GSP). www.genome.gov. Accessed July 24, 2020 at: www.genome.gov/sequencingcostsdata

7 Shay JW. Role of telomeres and telomerase in aging and cancer. Cancer Discov 2016;6(06):584-593
8 Horn S, Figl A, Rachakonda PS, et al. TERT promoter mutations in familial and sporadic melanoma. Science 2013;339(6122):959-961

9 Cancer Genome Atlas Research Network Electronic address: wheeler@bcm.edu Cancer Genome Atlas Research Network. Comprehensive and integrative genomic characterization of hepatocellular carcinoma. Cell 2017;169(07):1327-1341.e23

10 Cleary SP, Jeck WR, Zhao X, et al. Identification of driver genes in hepatocellular carcinoma by exome sequencing. Hepatology 2013;58(05):1693-1702

11 Guichard C, Amaddeo G, Imbeaud S, et al. Integrated analysis of somatic mutations and focal copy-number changes identifies key genes and pathways in hepatocellular carcinoma. Nat Genet 2012; 44(06):694-698

12 Schulze K, Imbeaud S, Letouzé E, et al. Exome sequencing of hepatocellular carcinomas identifies new mutational signatures and potential therapeutic targets. Nat Genet 2015;47(05):505-511

13 Totoki Y, Tatsuno K, Covington KR, et al. Trans-ancestry mutational landscape of hepatocellular carcinoma genomes. Nat Genet 2014;46(12):1267-1273

14 Dhanasekaran R, Nault J-C, Roberts LR, Zucman-Rossi J. Genomic medicine and implications for hepatocellular carcinoma prevention and therapy. Gastroenterology 2019;156(02):492-509

15 Tanaka S, Toh Y, Adachi E, Matsumata T, Mori R, Sugimachi K. Tumor progression in hepatocellular carcinoma may be mediated by p53 mutation. Cancer Res 1993;53(12):2884-2887

16 Hsu HC, Chiou TJ, Chen JY, Lee CS, Lee PH, Peng SY. Clonality and clonal evolution of hepatocellular carcinoma with multiple nodules. Hepatology 1991;13(05):923-928

17 Sirivatanauksorn Y, Sirivatanauksorn V, Bhattacharya S, et al. Evolution of genetic abnormalities in hepatocellular carcinomas demonstrated by DNA fingerprinting. J Pathol 1999;189(03): 344-350

18 Egeblad M, Nakasone ES, Werb Z. Tumors as organs: complex tissues that interface with the entire organism. Dev Cell 2010;18 (06):884-901

19 Ling S, Hu Z, Yang Z, et al. Extremely high genetic diversity in a single tumor points to prevalence of non-Darwinian cell evolution. Proc Natl Acad Sci U S A 2015;112(47):E6496-E6505

20 Shi J-Y, Xing Q Duan M, et al. Inferring the progression of multifocal liver cancer from spatial and temporal genomic heterogeneity. Oncotarget 2016;7(03):2867-2877

21 Xue R, Li R, Guo H, et al. Variable intra-tumor genomic heterogeneity of multiple lesions in patients with hepatocellular carcinoma. Gastroenterology 2016;150(04):998-1008

22 Buczak K, Ori A, Kirkpatrick JM, et al. Spatial tissue proteomics quantifies inter- and intratumor heterogeneity in hepatocellular carcinoma (HCC). Mol Cell Proteomics 2018;17(04):810-825

23 Kurebayashi Y, Ojima H, Tsujikawa H, et al. Landscape of immune microenvironment in hepatocellular carcinoma and its additional impact on histological and molecular classification. Hepatology 2018;68(03):1025-1041

24 Spitzer MH, Nolan GP. Mass cytometry: single cells, many features. Cell 2016;165(04):780-791

25 Goltsev Y, Samusik N, Kennedy-Darling J, et al. Deep profiling of mouse splenic architecture with CODEX multiplexed imaging. Cell 2018;174(04):968-981.e15

26 Mahe E, Pugh T, Kamel-Reid S. T cell clonality assessment: past, present and future. J Clin Pathol 2018;71(03):195-200

27 Losic B, Craig AJ, Villacorta-Martin C, et al. Intratumoral heterogeneity and clonal evolution in liver cancer. Nat Commun 2020;11 (01):291

28 Zhang Q, Lou Y, Yang J, et al. Integrated multiomic analysis reveals comprehensive tumour heterogeneity and novel immunophenotypic classification in hepatocellular carcinomas. Gut 2019;68 (11):2019-2031

29 Dong L-Q Peng LH, Ma LJ, et al. Heterogeneous immunogenomic features and distinct escape mechanisms in multifocal hepatocellular carcinoma. J Hepatol 2020;72(05):896-908 
30 Yin Z, Dong C, Jiang K, et al. Heterogeneity of cancer-associated fibroblasts and roles in the progression, prognosis, and therapy of hepatocellular carcinoma. J Hematol Oncol 2019;12(01):101

31 Lambrechts D, Wauters E, Boeckx B, et al. Phenotype molding of stromal cells in the lung tumor microenvironment. Nat Med 2018;24(08):1277-1289

32 Bartoschek M, Oskolkov N, Bocci M, et al. Spatially and functionally distinct subclasses of breast cancer-associated fibroblasts revealed by single cell RNA sequencing. Nat Commun 2018;9(01):5150

33 Sahai E, Astsaturov I, Cukierman E, et al. A framework for advancing our understanding of cancer-associated fibroblasts. Nat Rev Cancer 2020;20(03):174-186

34 Aird WC. Endothelial cell heterogeneity. Cold Spring Harb Perspect Med 2012;2(01):a006429

35 Ma L, Hernandez MO, Zhao Y, et al. Tumor cell biodiversity drives microenvironmental reprogramming in liver cancer. Cancer Cell 2019;36(04):418-430.e6

36 Tang X, Huang Y, Lei J, Luo H, Zhu X. The single-cell sequencing: new developments and medical applications. Cell Biosci 2019;9:53

37 Hou Y, Guo H, Cao C, et al. Single-cell triple omics sequencing reveals genetic, epigenetic, and transcriptomic heterogeneity in hepatocellular carcinomas. Cell Res 2016;26(03):304-319

38 Wang K, Sun D. Cancer stem cells of hepatocellular carcinoma. Oncotarget 2018;9(33):23306-23314

39 Ho DW-H, Tsui YM, Sze KM, et al. Single-cell transcriptomics reveals the landscape of intra-tumoral heterogeneity and stemness-related subpopulations in liver cancer. Cancer Lett 2019; 459:176-185

40 Zheng H, Pomyen Y, Hernandez MO, et al. Single-cell analysis reveals cancer stem cell heterogeneity in hepatocellular carcinoma. Hepatology 2018;68(01):127-140

41 Dandri M, Petersen J. Mechanism of hepatitis B virus persistence in hepatocytes and its carcinogenic potential. Clin Infect Dis 2016; 62(Suppl 4):S281-S288

42 Duan M, Hao J, Cui S, et al. Diverse modes of clonal evolution in HBV-related hepatocellular carcinoma revealed by single-cell genome sequencing. Cell Res 2018;28(03):359-373

43 Zhang Q He Y, Luo N, et al. Landscape and dynamics of single immune cells in hepatocellular carcinoma. Cell 2019;179(04): 829-845.e20

44 Zheng C, Zheng L, Yoo JK, et al. Landscape of infiltrating T cells in liver cancer revealed by single-cell sequencing. Cell 2017;169 (07):1342-1356.e16

45 Wang X, He Q, Shen H, Lu X-J, Sun B. Genetic and phenotypic difference in $\mathrm{CD}^{+} \mathrm{T}$ cell exhaustion between chronic hepatitis $\mathrm{B}$ infection and hepatocellular carcinoma. J Med Genet 2019;56(01):18-21

46 Alix-Panabières C. The future of liquid biopsy. Nature 2020;579 (7800):S9

47 Huang A, Zhang X, Zhou SL, et al. Detecting circulating tumor DNA in hepatocellular carcinoma patients using droplet digital PCR is feasible and reflects intratumoral heterogeneity. J Cancer 2016;7 (13):1907-1914

48 Cai Z-X, Chen G, Zeng YY, et al. Circulating tumor DNA profiling reveals clonal evolution and real-time disease progression in advanced hepatocellular carcinoma. Int J Cancer 2017;141(05): 977-985

49 Sun Y-F, Guo W, Xu Y, et al. Circulating tumor cells from different vascular sites exhibit spatial heterogeneity in epithelial and mesenchymal composition and distinct clinical significance in hepatocellular carcinoma. Clin Cancer Res 2018;24(03):547-559

50 Huang A, Zhao X, Yang XR, et al. Circumventing intratumoral heterogeneity to identify potential therapeutic targets in hepatocellular carcinoma. J Hepatol 2017;67(02):293-301

51 Mitra A, Mishra L, Li S. Technologies for deriving primary tumor cells for use in personalized cancer therapy. Trends Biotechnol 2013;31(06):347-354

52 Gao Q Wang ZC, Duan M, et al. Cell culture system for analysis of genetic heterogeneity within hepatocellular carcinomas and response to pharmacologic agents. Gastroenterology 2017;152 (01):232-242.e4

53 Drost J, Clevers H. Organoids in cancer research. Nat Rev Cancer 2018;18(07):407-418

54 Li L, Knutsdottir H, Hui K, et al. Human primary liver cancer organoids reveal intratumor and interpatient drug response heterogeneity. JCI Insight 2019;4(02):e121490

$55 \mathrm{Hu}$ B, Li H, Guo W, et al. Establishment of a hepatocellular carcinoma patient-derived xenograft platform and its application in biomarker identification. Int J Cancer 2020;146(06):1606-1617

56 Blumer T, Fofana I, Matter MS, et al. Hepatocellular carcinoma xenografts established from needle biopsies preserve the characteristics of the originating tumors. Hepatol Commun 2019;3(07): 971-986

57 Zhao Y, Shuen TWH, Toh TB, et al. Development of a new patientderived xenograft humanised mouse model to study humanspecific tumour microenvironment and immunotherapy. Gut 2018;67(10):1845-1854

58 Sato K, Niida A, Masuda T, et al. Multiregion genomic analysis of serially transplanted patient-derived xenograft tumors. Cancer Genomics Proteomics 2019;16(01):21-27

59 Rajaram S, Roth MA, Malato J, et al. A multi-modal data resource for investigating topographic heterogeneity in patient-derived xenograft tumors. Sci Data 2019;6(01):253

60 Dagogo-Jack I, Shaw AT. Tumour heterogeneity and resistance to cancer therapies. Nat Rev Clin Oncol 2018;15(02):81-94

61 Craig AJ, Labgaa I, Villacorta-Martin C, Ningarhari M, Villanueva A. Tumor heterogeneity and resistance to targeted therapies in hepatocellular carcinoma. Resistance Targeted Anti-Cancer Therapeutics 2017:1-24

62 Turner NC, Reis-Filho JS. Genetic heterogeneity and cancer drug resistance. Lancet Oncol 2012;13(04):e178-e185 